\title{
FILAMENTS EVOLUTION IN EXTENDED RADIO SOURCES
}

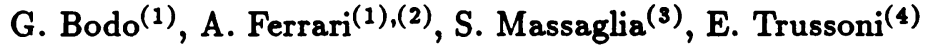 \\ (1) Osservatorio Astronomico di Torino, Pino Torinese, Italy \\ (2) Istituto di Fisica Matematica, Università di Torino, Italy \\ (3) Istituto di Fisica Generale, Università di Torino, Italy \\ (4) Istituto di Cosmo-geofisica del C.N.R., Torino, Italy
}

It has been recently shown (Bodo et al 1989) that filamentary structures observed in some extended radio sources (e.g. Cygnus A and M 87) can be related with thermal instabilities arising in the gas with a synchrotron radiating relativistic component. In particular is has been shown that, with the typical parameters of radio sources, two kind of unstable modes can develop on time scale comparable with the synchrotron cooling time: condensation stationary modes and travelling slow MHD waves. Both these kind of perturbations should lead to the formation of structures basically parallel to the magnetic field direction, as observed, however their general evolution, in particular if they will lead to the formation of dark or bright structures, cannot be extrapolated from the linear perturbative analysis.

For this purpose we can study the evolution of the emissivity $\mathcal{L}(t) \propto$ $B(t)^{2} N(t) \bar{\gamma}(t)$, where $B$ is the magnetic field, $N$ the density of relativistic particles, and $\bar{\gamma}$ the particles average energy. As the general behaviour of these quantities beyond the linear regime is unknown in relation with the possible evolution of the condensation mode we shall assume a total pressure equilibrium. is given by:

The evolution of the spectrum of the particles against synchrotron losses

$$
\frac{\partial N}{\partial t}=\beta \frac{\partial}{\partial \gamma}\left(\gamma^{2} N\right) \quad \rightarrow \quad \frac{\partial N}{\partial \xi}=\frac{\partial}{\partial \gamma}\left(\gamma^{2} N\right),
$$

with $\beta(t)=b B(t)^{2}, d \gamma / d t=-\beta \gamma^{2}$, and where the differential relationship $d \xi=\beta d t$ holds. The solution of Eq. (1) is

$$
N(\gamma, \xi)=C_{0} \gamma^{-\Gamma}(1-\xi \gamma)^{\Gamma-2}, \quad C_{0} \int_{\gamma_{\min }}^{\gamma_{\max }} N(\gamma, 0) d \gamma=1
$$

while the evolution of the average energy is:

$$
\bar{\gamma}(\xi)=\int_{\gamma_{1}(\xi)}^{\gamma_{2}(\xi)} N(\gamma, \xi) \gamma d \gamma, \quad \gamma_{1}=\frac{\gamma_{\min }}{1+\xi \gamma_{\min }}, \quad \gamma_{2}=\frac{\gamma_{\max }}{1+\xi \gamma_{\max }} .
$$

The magnetic field evolution is provided by the balance of the total pressure and the frozen in condition in a quasi-static medium:

$$
\frac{B^{2}(\xi)}{8 \pi}+P(\xi)=A_{0} \simeq \text { constant }, \quad \frac{B(\xi)}{N(\xi)}=A_{1} \simeq \text { constant } .
$$


From these relations and from the relativistic pressure for the relativistic component $P(\xi)=\frac{1}{3} N(\xi) \bar{\gamma}(\xi) m_{e} c^{2}$ we obtain:

$$
B(\xi)=\frac{4 \pi}{3} \frac{\bar{\gamma}}{A_{1}}\left[\left(1+\frac{9 A_{0} A_{1}^{2}}{2 \pi \bar{\gamma}^{2}}\right)^{1 / 2}-1\right] .
$$

From previous equations we can finally calculate the emissivity at a time $t$ :

$$
\mathcal{L}(\xi)=C_{0} b \int_{\gamma_{1}(\xi)}^{\gamma_{2}(\xi)} B^{2}(\xi) \gamma^{2} N(\xi) \gamma^{-\Gamma}(1-\xi \gamma)^{\Gamma-2} d \gamma, \quad t=\int_{0}^{\xi} \frac{d \xi^{\prime}}{b B^{2}\left(\xi^{\prime}\right)}
$$

In Fig. 1 we plot the emissivity as a function of time for $\gamma_{\min }=10^{3}$ and $\gamma_{\max }=$ $10 \gamma_{\min }$, and different values of $\Gamma$ and $B(0)=10^{-4} \mathrm{G}$ (with radiation emission in the range $0.04-4 \mathrm{GHz}$ ). The emissivity increases (up to a factor $\sim 2$ ) in $\sim 10^{6}$ years, which is $\sim$ one synchrotron time for electrons with $\gamma \approx 10^{3}$. The filaments formed in such conditions would actually appear bright to observations, and this is in agreement with the linear analysis that predicts instability with the assumed previous parameters. These rough evaluations seem to indicate that, in a reasonable range of physical parameters, filaments can undergo an initial transient phase of luminosity increase, on time comparable with the synchrotron cooling time scale, before evolving into cool condensed structures.

\section{REFERENCES}

Bodo,G., Ferrari,A., Massaglia,S., Trussoni, E., 1989, M.N.R.A.S., submitted

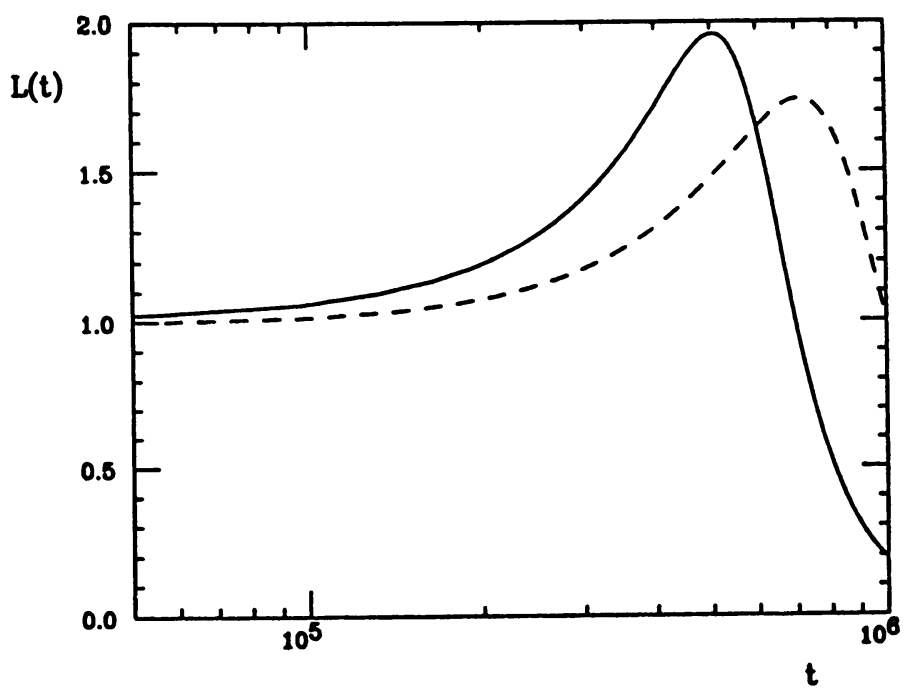

Figure 1: Temporal evolution of $L(t)=\mathcal{L}(t) / \mathcal{L}(0)$ ( $t$ in years) for $\Gamma=2$ (full line) and $\Gamma=3$ (dashed line). 